\title{
The Computational Model of Spring back Accuracy on Steel Bar Bending Hoop Stirrup
}

\author{
Bianjing $\mathrm{Li}^{1}$ and Zhiliang $\mathrm{Liu}^{2}$ \\ ${ }^{1}$ School of Continuing Education, Yanshan University, Qinhuangdao 066004, Hebei, China \\ ${ }^{2}$ National Engineering Research Center for Equipment and Technology of Cold Strip Rolling, \\ Yanshan University, Qinhuangdao 066004,Hebei,China
}

Keywords: Computational model; Spring-back angle; Finite element simulation

\begin{abstract}
The hooked steel will spring back under the affection of residual stress, in order to improve the precision of the hoop, spring back angle should be taken into consideration. According to spring-back theory, considering the work hardening of steel under the straightening rollers, the relation was deduced between hoop angle and spring-back angle. Based on the hoop-bending machine with profile modeling, the relation was deduced between rotation of rotary-table and hoop angle, and give reference to the processing parameter during the NC machining. Establish the model of hoop based on the finite element analysis software MARC, analyses the influence on spring-back angle under different hoop speed and different radius of gyration. The experiment on hoop-bending machine verified the result of finite element simulation.
\end{abstract}

\section{Introduction}

Steel is widely used in architecture, civil engineering and other fields, plays an important role in the national infrastructure construction. In recent years, new III grade steel is widely used for its high strength, good extensibility. The strength of new III grade steel is about $20 \%$ higher than traditional steel, which has put forward higher requirements to the steel processing industry. it should be hooped during steel processing process, at the same time there is a region of plastic deformation and elastic deformation in the steel cross section area[1]. Steel have an angle after unloading of residual stress, so that the hoop angle is too large .In order to improve the precision of hoop, the spring-back is usually controlled by the over bending method[2] in the production. Over bending method is a method that bases on the size of spring-back value after unloading bending parts, then increasing the hoop stroke of hoop-bending shaft reasonably, in order to achieve the predetermined angle after spring back. If over bending method is used, spring-back angle should be predicted before hoops steel, so that processing parameter can be settled reasonably on hoop bending machine.

For bending spring back problems, foreign and domestic scholars have conducted massive research mainly by using finite element simulation method, theoretical analysis method and experimental method[3-5]. The finite element simulation method is widely used because of its merits of low cost and high efficiency. Assumptions were introduced to simplify model in the simulation of bending spring back process by using finite element method. The simulation results are verified by hoop bending experiment to ensure the reliability of simulated results.

\section{Working Principle of Fop Bending Machine}

Steel enter the hoop bending mechanism from the center hole of the lower reamer block after straightening. Hoop bending rotary-table structure as shown in Fig. 1, hoop bending shaft and rotary-table is concentric but uncoaxiality, the hoop-bending shaft is fixed on the rotary-table, rotary-table can rotate by the spindle driven. When it hoops ,the steel is forced to bend by the hoop 
bending shaft, after reaching a predetermined angle, rotary-table back switching to the initial position, hoop action is completed. Steel will have a bending process in rotary process of rotary-table, the angle after spring back is the final hoop angle.

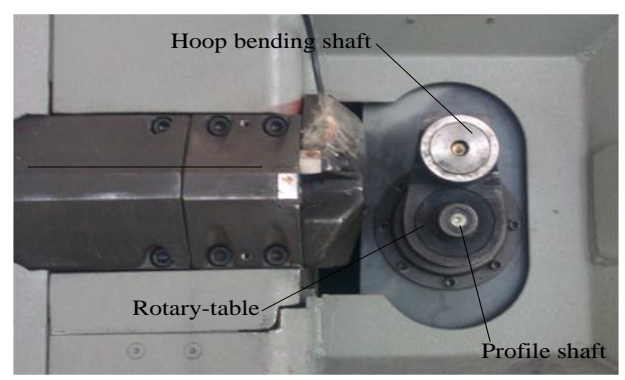

Figure 1. The structure of hoop-bending machine

\section{The Mathematical Model of Bending Hoop}

Fig. 2 is a schematic diagram of the process of spring back, the hooped is the solid line, the steel of spring back is dotted line, Under the action of hoop bending shaft, turning angle is $\theta_{1}$, the radius of curvature of the steel is $R_{3}$, the hoop angle is $\theta_{3}$. The hoop bending shaft reset when the hoop is completed, under the effect of the internal residual stress ,the steel will rebound an angle that is $\Delta \theta$.After spring back ,the angle of steel becomes $\theta_{2}$, the radius of curvature of teal becomes into $R_{4}$, hoop angle becomes into $\theta_{4}$.

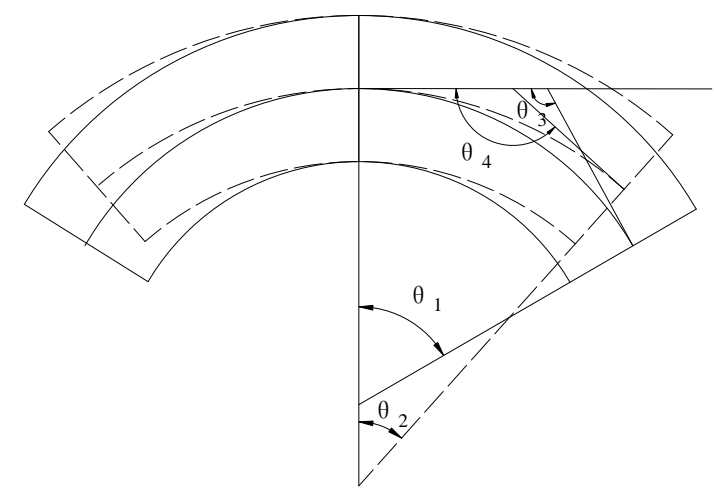

Figure 2. The process of spring back

The process of hoop belongs to the lactic-plastic bending, according to the principle of invariance of neutral layer[6]

$\mathbf{R} Q=\mathbf{R}_{4} Q_{2}$

According to the last type:

$$
\begin{aligned}
& Q=\frac{\mathrm{R}_{4}}{\mathrm{R}_{3}} Q=\frac{\mathrm{R}}{\mathrm{R}}(\pi-Q) \\
& \qquad Q Q G(\pi Q Q)+1
\end{aligned}
$$

The machining process of steel include straightening, bending hoop, cut off. Steel will produce elastic-plastic deformation in repeatedly under the action of straightening rollers, it will produce work hardening. Therefore the effect of work hardening on spring back angle should be taken into 
account, when calculate the bending spring back of steel.

The curvature equation considering the strengthening [10]

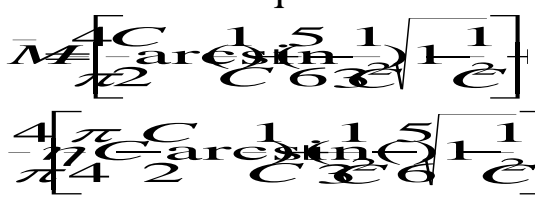

In the formula , $\mathrm{C}$ is the relative total curvature, $\eta$ is metal hardening coefficient. Steel is approximately flat after straightening, $C_{0}=0$.

According to straightening theory:

$$
\begin{aligned}
& C C_{w}+C_{\theta} \\
& C_{c}=C_{w}-C_{1}
\end{aligned}
$$

$C_{\mathrm{f}}=\bar{M}$

$C_{f}$ is relative curvature of spring back, $C_{c}$ is relative residual curvature, $C_{w}$ is reverse curvature.

Collating the curvature equation of the bar:

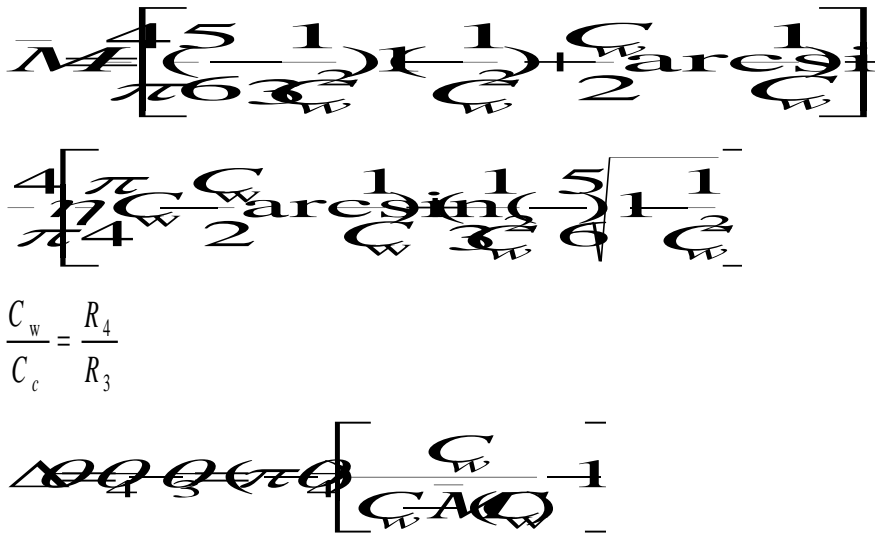

In the above formula, when the profile shaft radius is certainly, $C_{w}$ is a certain value, according to the hoop angle $\theta_{4}$ can calculate the spring back angle of steel.

Fig. 3 shows a schematic diagram of hoop bending, $d$ is the diameter of steel, $R$ is the radius of gyration, $R_{1}$ is the radius of hoop bending shaft, $R_{2}$ is radius of the profile shaft, $\alpha$ is the angle of rotary-table, simultaneously is the slope angle of steel, $\theta$ is the hoop angle

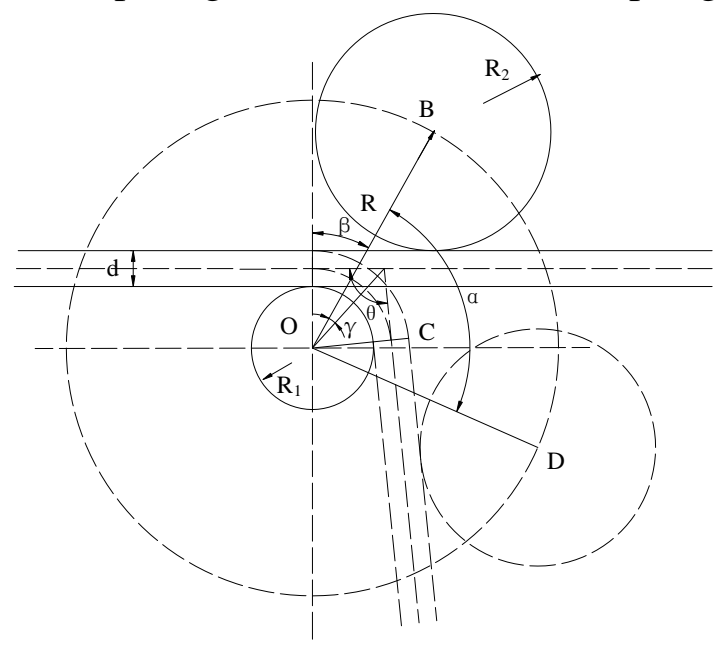

Figure 3. The parameter of hoop-bending rotary table 
According to the geometric diagram:

$$
\left\{\begin{array}{l}
\frac{\left(R_{1}+d\right)}{\cos \beta}+\frac{R_{2}}{\cos \beta}=R \\
\gamma=\frac{\pi-\theta}{2}-\beta \\
\angle C O D=\alpha-\gamma-\frac{\pi-\theta}{2} \\
\frac{R_{1}+d}{\cos \angle C O D}+\frac{R_{2}}{\cos \angle C O D}=R
\end{array}\right.
$$

The equations can obtained the relationship between hoop angle and slope of hoop bending shaft $\theta=\pi$

\section{Finite Element Simulation of the Bending Hoop}

In order to analyze the effect of steel hoop speed on spring back angle, refer to table 2 the model of hoop bending is established and the simulation parameters is settled. Bending spring back process were simulated when hoop bending shaft angular velocity $\omega$ is $0.3 \mathrm{rad} / \mathrm{s}, 3 \mathrm{rad} / \mathrm{s}, 30 \mathrm{rad} / \mathrm{s}$.

Table 1 The simulation parameters

\begin{tabular}{cccc}
\hline Yield limit of steel & Profiled shaft radius & Radius of gyration & Radius of steel \\
\hline $400 \mathrm{MPa}$ & $35 \mathrm{~mm}$ & $70 \mathrm{~mm}$ & $10 \mathrm{~mm}$ \\
\hline
\end{tabular}

Fig. 4 is the residual stress echogram of steel in longitudinal section when the steel turn an angle $\alpha=100^{\circ}$ and hoop bending shaft will reverse rotation. When the hoop bending has ended, the distribution of internal residual stress is not uniform, the lateral steel is stretched, internal steel is compressed. The steel will occur spring back under internal residual stress after the external force is removed. According to the echogram : When the hoop speed is different, steel residual stress values and distribution is basically same. Thus the difference of steel spring back angles is small, so it can be approximated that there is no change.

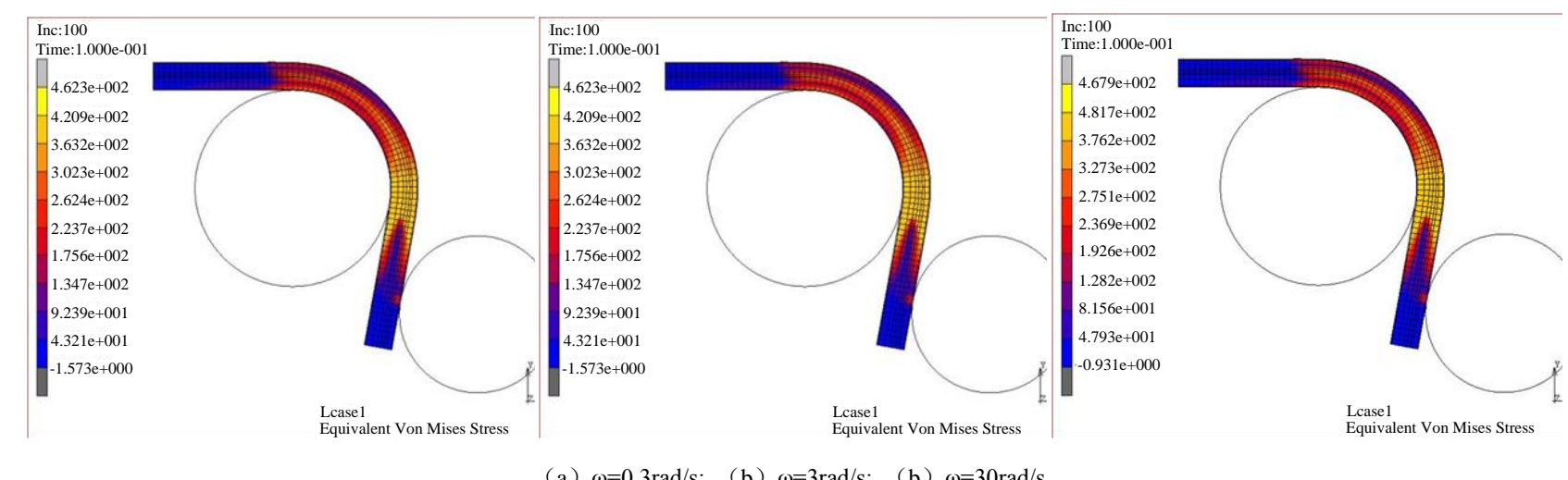

Figure 4. The stress-strain diagram of steel 
Fig. 5 shows the different hoop bending shaft of the radius of gyration corresponds to the spring back angle of the steel, we can see that, when the other conditions were the same, the spring-back angle increase with radius of gyration increasing .

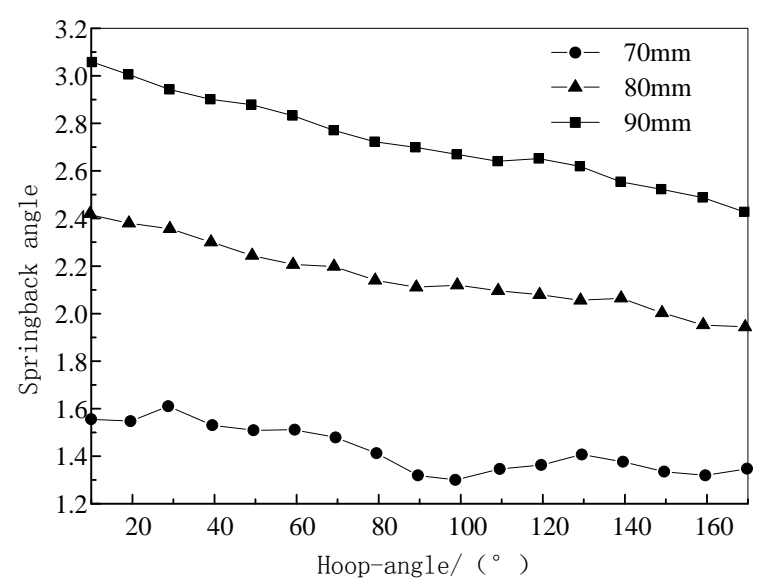

Figure 5. The numerical of spring-back angle

\section{Conclusions}

According to the theory of bending spring back and considering the effect of work hardening on material properties, the relation was deduced between the hoop angle and the spring back angle, the relation was deduced between rotation of rotary-table and hoop angle, and give reference to the processing parameter during the $\mathrm{NC}$ machining

\section{References}

[1] Xue Jun an, Hu Xian lei, Liu Xiang hua etc. Mathematical Model of Elastic Plastic Bending for Roller Leveling [J]. Journal of Iron and Steel Research,2008, 20(11): 33.

[2] GU Rui jie, Yang He, Zhan Mei. A Review of the Research on Spring back in Bendin g [J].MECHANICAL SCIENCE AND TECHNOLOGY,2005, 24(6): 653-658.

[3] Huawei Zhang, Li-zhong LIU, Ping HU etc. Numerical Simulation and Experimental Investigation of Spring back in U-Channel Forming of Tailor Rolled Blank[J]. Journal of Iron and Steel Research,2012,19(9): 08-12.

[4] Wang Xiu ling, causes bending spring back of sheet and method for reducing rebound [j]. electric manufacturing,2014,4: 66-67. 